\title{
Case Based Reasoning with Expert System and Swarm Intelligence to determine Energy Reduction in Buildings Energy Management
}

\author{
Ricardo Faia ${ }^{1}$, Tiago Pinto ${ }^{1,2 *}$, Omid Abrishambaf ${ }^{1}$, Filipe Fernandes ${ }^{1}$, \\ Zita Vale ${ }^{1}$ and Juan Manuel Corchado ${ }^{2}$ \\ ${ }^{1}$ GECAD - Research Group on Intelligent Engineering and Computing for Advanced Innovation and \\ Development of the Polytechnic of Porto (ISEP/IPP) \\ R. Dr. António Bernardino de Almeida, 431, 4200-072 Porto, Portugal \\ ${ }^{2}$ BISITE Research Centre, University of Salamanca \\ Calle Espejo, 12, 37007 Salamanca, Spain
}

\section{ABSTRACT}

This paper proposes a novel Case Based Reasoning (CBR) application for intelligent management of energy resources in residential buildings. The proposed CBR approach enables analyzing the history of previous cases of energy reduction in buildings, and using them to provide a suggestion on the ideal level of energy reduction that should be applied in the consumption of houses. The innovations of the proposed CBR model are the application of the $k$-Nearest Neighbors algorithm (k-NN) clustering algorithm to identify similar past cases, the adaptation of Particle Swarm Optimization (PSO) meta-heuristic optimization method to optimize the choice of the variables that characterize each case, and the development of expert systems to adapt and refine the final solution. A case study is presented, which considers a knowledge base containing a set of scenarios obtained from the consumption of a residential building. In order to provide a response for a new case, the proposed CBR application selects the most similar cases and elaborates a response, which is provided to the SCADA House Intelligent Management (SHIM) system as input data. SHIM uses this specification to determine the loads that should be reduced in order to fulfill the reduction suggested by the CBR approach. Results show that the proposed approach is capable of suggesting the most adequate levels of reduction for the considered house, without compromising the comfort of the users.

KEYWORDS: Artificial Intelligence, Case Based Reasoning, Demand Response, Energy Efficiency, Intelligent House Energy Management

\footnotetext{
* Corresponding author: Tiago Pinto is with GECAD - Research Group on Intelligent Engineering and Computing for Advanced Innovation and Development, R. Dr. António Bernardino de Almeida, 431, 4200-072 Porto, Portugal; Tel.: +351 22 8340500; Fax: +351 22 8321159, WebSite: http://www.gecad.isep.ipp.pt / Email: tmcfp@isep.ipp.pt
} 


\section{Introduction}

In the European Union (EU), buildings are responsible for $40 \%$ of energy consumption and $36 \%$ of $\mathrm{CO}_{2}$ emissions. These quantities are due to the fact that most of the buildings in EU are outdated (35\% are over than 50 years old) [10]. As this sector is considered to be expanded, the increment of energy consumption obligates the EU to reduce it and utilize the renewable energy resources in the demand side [16]. These measures were taken to allow the EU to comply with the Kyoto Protocol to the United Nations Framework Convention on Climate Change, which aims to reduce global greenhouse gas emissions by at least $20 \%$ belong 1990 levels to the year 2020 [8]. According to the data provided by European Commission, in each country, the electricity consumption in the service and household sectors are higher than the consumption in the transport or industrial services section [2].

Generally, the consumption in buildings is due to people spending most of their time indoors, whether for housing or work. Due to the relationship between the productivity and comfort at work, the operation costs of an office building are directly linked to the workers' income [33]. In this sense, the energy consumption and the conditions of environmental comfort are, in most cases, in conflict with each other [6]. Due to the existing scenario of energy consumption in buildings, combined with the fact that construction activity accounts for about one-eighth of the European economy, the EU has released the Energy Performance of Buildings Directive (EPBD), which concerns the energy use in buildings. The EU calls on member states to introduce stricter regulations on the efficient use of energy in buildings [9].

The Smart Home concept is defined as a residence equipped with sensors and possibly actuators to collect data and send controlling commands, according to the activities and expectations of the occupants [3], which make it possible to develop a Building Energy Management System (BEMS) [20]. BEMS is considered as a system that determines energy management strategies in order to change the way of energy consuming and producing. This enables the occupants to achieve better energy performance and comfort [22].

In accordance with the requirements imposed by the EU for reducing $\mathrm{CO}_{2}$ emissions, where buildings are considered to be the casters of most of these emissions, energy efficiency should be increased, by decreasing the consumption of unnecessary power; and buildings should be able to respond to demand response events, where some need for reduction can be asked in specific times (e.g. during times when renewable based generation in lower). Although the existing BEMS are able to provide some contribution in this direction, there 
is a significant difficulty in identifying the exact values of consumption reduction that could be asked or applied to each consumer.

In [30], authors present a mathematical model for the optimal energy management of residential buildings and propose a centralised energy management system. The mathematical model was constructed including the model of each component and their physical constraints, parameter settings, external information, and user preferences to generate optimal decisions. By requiring the exact information of all parameters, including the models of all components, user preferences and external factors, which are usually variable, e.g. temperature, luminosity, this model becomes limited in terms of application, as it can only be applied in contexts in which all the information is available. Using the approach proposed in the current paper, the Case Based Reasoning (CBR) approach uses historical data from past knowledge to learn from past experiences, thereby becoming much more open to a wide set of new application scenarios. The execution time and simplicity of implementation (as the proposed model does not require the mathematical formulation of all components and settings) are other relevant advantages of the proposed model.

An application of CBR in the context of buildings, more precisely in green buildings, can be seen in [37]. In this publication, authors developed a model using CBR and text mining, which tries to take advantage of the evaluations of green buildings and their conclusions and solution, to learn lesson to be applied in new cases, with the objective of predicting if the new buildings will be successful in their evaluation. The use of CBR in buildings has been widely used for predicting consumption as can be observed in the following sources [26,36]. In the current paper, on the other hand, CBR is used to support decisions on how much electricity can be reduced in a house.

This paper addresses the described problem, and overcomes the identified limitations by proposing a CBR methodology that determines the level of instantaneous reduction in a building that could be applied without compromising the comfort of the users. The proposed CBR model considers a database of registered past scenarios referring to the same building, and generates a reduction value based on the existing cases. Firstly, the k Nearnest Neighbors (k-NN) clustering algortihm [5] is applied to identify the most similar cases to the current one. After the similar cases are identified, an optimization process using Particle Swarm Optimization (PSO) [15] is applied to optimize the weights attributed to each variable that characterizes each case, in order to reach the optimal combination of the similar cases with the aim of achieving a solution for the new case. 
Finally, the achieved solution is refined by means of an expert system [35]. After the final solution is achieved, the solution is sent to the SCADA House Intelligent Management (SHIM) [13], which is a BEMS in the residential context, which will decide which loads should be reduced based on the output results proposed by the CBR approach and taking into account the current context and the users' comfort.

This article is divided into 6 sections. After this introductory section, where the contextualization of the problem is described, section 2 provides an overview on the CBR process, considering all the stages related to the common CBR cycle. Section 3 provides a description of SHIM, while section 4 presents the proposed model, including a description of the applied techniques. Section 5 describes the case study and presents and discusses the achieved results. Finally, the main conclusions of the work are provided in section 6.

\section{Case Based Reasoning}

The CBR methodology is defined as an intelligent technique to learn CBR biases in the past for solving new problems, and also capture new knowledge/experience to apply it in the future [17]. Research in cognitive science on human memory was the main driver for the development of CBR, as it tried to portray how the human brain reacts when situations that require reasoning emerge [29]. As an example: imagine that the hand of a human is in the eminence of meeting a flame, if the human brain still did not experience that situation, the human will contact the flame and he/she will suffer the consequences. On the other hand, if the brain already has the information that this action is harmful to him/her, there will not be a contact with the flame. This example clarifies the use of past knowledge to act on future. Similarly, the CBR is based on memory, which enables the human to use the remembered solutions of the problems, as the starting point of new solutions of problems, contrarily to most of the problem-solving methodologies in Artificial Intelligence (AI) [18].

It can be considered that the CBR is a subfield of the learning machine since it is mostly driven by the machine learning the community. Thus, the CBR assumes itself as a paradigm of machine learning that allows sustained learning by updating the case base after a problem has been solved. Learning in the CBR occurs as a natural result of solving the problem. When a problem is solved successfully, the experience is maintained to solve similar problems in the future. CBR learning process requires a well-developed set of methods in order to be able to extract relevant knowledge from experience, to integrate a case into an existing knowledge structure, and to index the case for later correspondence with similar cases [1]. 
Janet Kololner developed the first system that can be called CBR, entitled CYRUS, which is basically a question and answer system with knowledge of the various trips and meetings of former Secretary of State Cyrus Vance [29]. The PROTOS system is also a tool based on the CBR and used in classification task. This system was developed by Bruce Porter and his team [2].

Figure 1 illustrates the operating principle of a CBR system. After the presentation of a problem, the retrive process of one or more of previously exprimented cases is undertaken. Afterwards, CBR reuses the case(s) in a specific way, depending on the problem in hands, and reviews the solution based on the reuse of a previous case. Finally, the new experience is retained by incorporating it in the knowledge base.

As Figure 1 illustrates, there are four main tasks in a CBR cycle: Retrieve, Reuse, Revise, and Retain. These four tasks are described as follows:

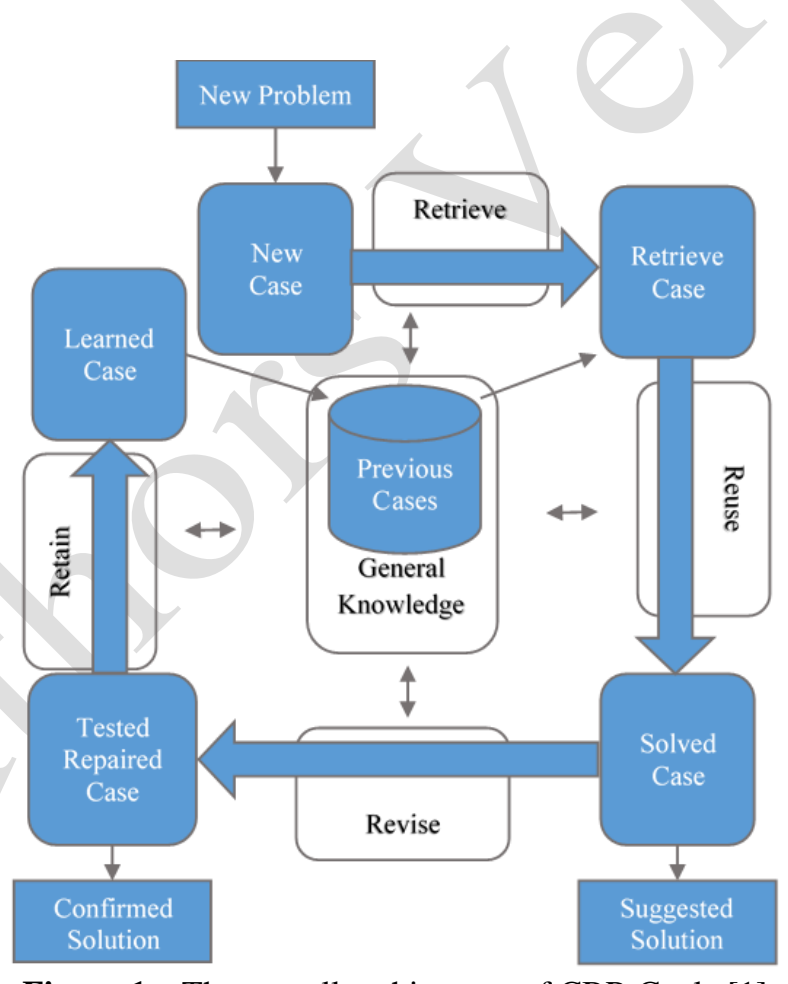

\subsection{Retrieve}

Retrieval is a very important step in the CBR cycle. As one can see in Figure 1, in this step the new case and the retrieved case are compared, so that the system can select the most similar cases to the new problem. If the best (most similar) cases are not identified in this step, the rest of the CBR process will not provide any useful results or information. For this step, classical methods such as k-NN [5] or Fish and Shrink [28], or more 
elaborate methods, such as artificial neural networks [14] or genetic algorithms [31] can be useful. Furthermore, there are some statistical methods to recover the best cases of the case base [4]. In [25], a method of extracting the similar cases called Statistical CBR is proposed, which recovers the optimal number of neighbors based on their probabilistic similarity.

\subsection{Reuse}

The reuse task in the CBR cycle is responsible for proposing a solution to the new problem [27]. This solution is based on the case(s) collected from the retrieve process. As can be seen in the Figure 1, this is the second stage of the cycle. In many cases, this process becomes easy to execute since the solution of the new problem can be the same as the recovered solution, and will be unchanged. This situation happens in classification tasks, where the most similar recovered case (if they are sufficiently similar) is susceptible and contains an appropriate solution. When the new case and the retrieved cases present significant differences, the process becomes more difficult. In this case, it is necessary to adapt the solutions of the retrieve case to obtain a new solution [19].

There are problems in which it is even possible to perform the adaptation of the solution if the solution to the new case does not exist in the case base, as is the case of design, configuration and planning problems. According to Janet Kolodner [17], the adaptation can be clasiffied in two ways: substitution and adaptation. Substitution simply reestablishes some parts of the recovered solution, and adaptation is the transformation where the solution structure changes. Graza \& Maher [32] proposed the use of evolutionary methods for the process of adaptation for the new solution. In this case, the solutions of the retrieved cases become the initial population for a genetic algorithm, where mutation and crossover operators are used to generate new solutions for the population.

\subsection{Revise}

As it is clear from Figure 1, the revise process starts when the reuse process is finished. The review aims to assess the applicability of the proposed solution. The evaluation of the solution is often done in simulations, as it would be very risky to make the evaluation in the real world, (e.g, in the area of the medicine, it could injure the human). On the other hand, sometimes the simulation can not represent the reality and can neglect important aspects. This situation can be compared to an existing problem in AI called a "frame problem", which mentions that one can never completely formulate all possible factors that can occur in the real world [21]. 


\subsection{Retain}

The retention task is the last step to be carried out in the CBR classic cycle, in which the recently solved case is incorporated into the knowledge base of the system. In most CBR systems, the specification of the problem and the final solution are recorded, assuming that the result was successful. But there are more complex systems that hold other types of data that record a much deeper representation of the problem in order to solve the process that led to the specific solution. In the retain process, Veloso \& Carbonell [34], not only store the final solution, but also save knowledge structures that describe how a particular solution was constructed in order to provide a path of decision-making processes that led to a particular solution.

\section{SCADA House Intelligent Management (SHIM)}

The SHIM system is a testbed platform for power consumption optimization and learning model application. One of the main objectives of SHIM is to adjust the system with the user preferences and the environmental parameters via learning algorithms. For this purpose, a new learning model has been developed in order to enable the SHIM platform to optimze the use of energy while it is required.

\subsection{System Architecture}

The overall view of the SHIM platform is illustrated in Figure 2. The main objective of SHIM is to simulate, examine, and survey the new methodologies and algorithms, which are going to be utilized in house or building management. This platform includes several real hardware equipment in order to provide a realistic simulation platform, namely several types of loads, Photovoltaic (PV) and wind generation as mini and micro distributed generation (DG), and energy storage systems to simulate electric vehicles.

The SHIM platform has been developed in the Institute of Engineering - Polytechnic of Porto (ISEP/IPP). In fact, SHIM is a section of a large simulation platform called Multi-Agent Smart Grid Simulation Platform (MASGriP), which is test platform for simulate and evaluating the competitive enviornment in future power systems [24]. SHIM is capable of controling real and virtual loads to ensure the simulation of complex scenarios. In the virtual loads, the technical and realistic parameters of the real ones have been applied. As it can be seen on Figure 2, the platform consists of three main groups: the Data Acquisition, Actuators, and Intelligent Applications. All of the operations regarding the learning algorithms located on Intelligent Application category. More information about the structure of SHIM platform are available in [13]. 


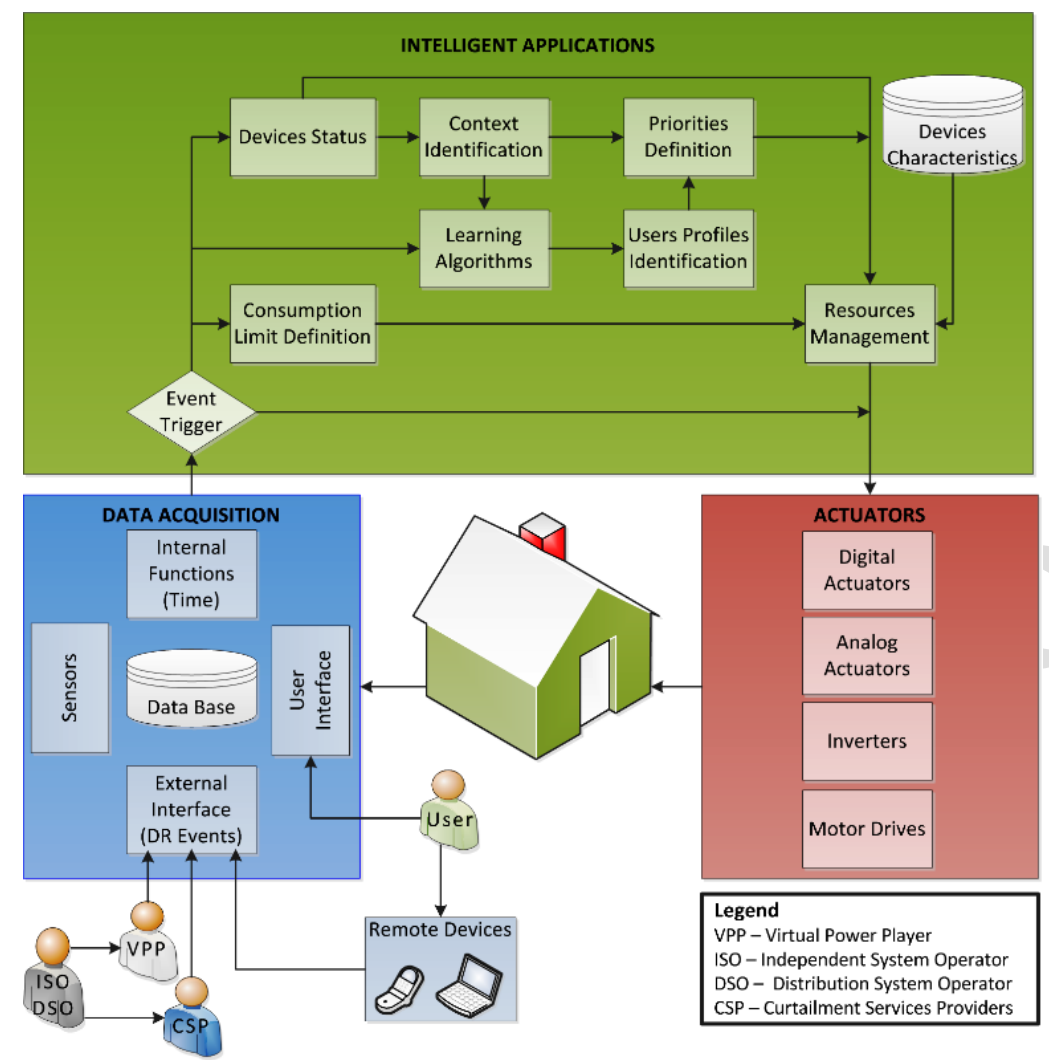

Figure 2 - SHIM platform overview [13].

\subsection{Optimization algorithm}

There is an optimization algorithm developed for SHIM in order to minimize the impact of power curtailments on user comfort and preferences. This algorithm consists of two regulation variables: regulation up and down, which are responsible for optimization feasibility. This means that if the regulation down is equal to zero, the optimization has achieved an adequate solution, and if it is greater than zero, the optimization has gained a solution with higher power consumption comparing with power limit.

SHIM takes into the account the comfort level, and the user interaction in different types of the events. The objective function used in the algorithm is shown in equation (1):

$$
\begin{aligned}
& \text { Minimize } f=\min \\
& \left\{\begin{array}{l}
\sum_{\text {Load }=1}^{n \text { Load }} \lambda_{\text {Load }} \times P_{\text {Load }}+\lambda_{\text {Grid }} \times P_{\text {Grid }}+\lambda_{\text {Down }} \times \operatorname{Reg}_{\text {Down }} \\
-\sum_{D G=1}^{n D G} \lambda_{D G} \times P_{D G}-\lambda_{U P} \times \operatorname{Reg}_{U p}
\end{array}\right\}
\end{aligned}
$$


Where

$\begin{array}{llll}\lambda_{D G} & \text { DG priority } & \text { Load } & \text { Load index (ID) } \\ \lambda_{\text {Down }} & \text { Regulation down priority } & n L o a d & \text { Maximum number of loads } \\ \lambda_{\text {Grid }} & \text { Grid priority } & P_{D G} & \text { Power generation of DG [W] } \\ \lambda_{\text {Load }} & \text { Load priority } & P_{\text {Grid }} & \text { Power injection in the grid [W] } \\ \lambda_{U p} & \text { Regulation up priority } & P_{L o a d} & \text { Power consumption of load [W] } \\ D G & \text { DG index (ID) } & \operatorname{Reg}_{\text {Down }} & \text { Power regulation down [W] } \\ n D G & \text { Maximum number of DG } & \operatorname{Reg}_{U p} & \text { Power regulation up [W] }\end{array}$

The main purpose of optimization algorithm is to guarantee the power consumption limit, represented by $P_{\text {Limit }}$ in the objective function. $\lambda_{\text {Loa }}$ represents the resource priority factor, which is varies from 0 (lowest priority resources) to 10 (highest priority resources). More detailed information regarding the formulation and its nomenclature can be found in [13].

\section{Proposed model}

The model proposed in this paper is represented through Figure 3. Figure 3 shows the connection between SHIM and the CBR approach. The proposed methodology aims to reduce the instant consumption of buildings.

As can be observed by Figure 3, the data collected by the measurement units constitute knowledge for the model. This knowledge is used by the CBR in order to reach a reduction value, assimilating the knowledge of the past situations with the current state that is registered in the building. After the reduction value is generated, it can be sent to SHIM, which optimizes the consumption and generation according to the target reduction value. All cases are saved in a data base so that they can be acceded in the future. An overview of the proposed CBR approach is presented in Figure 4. 


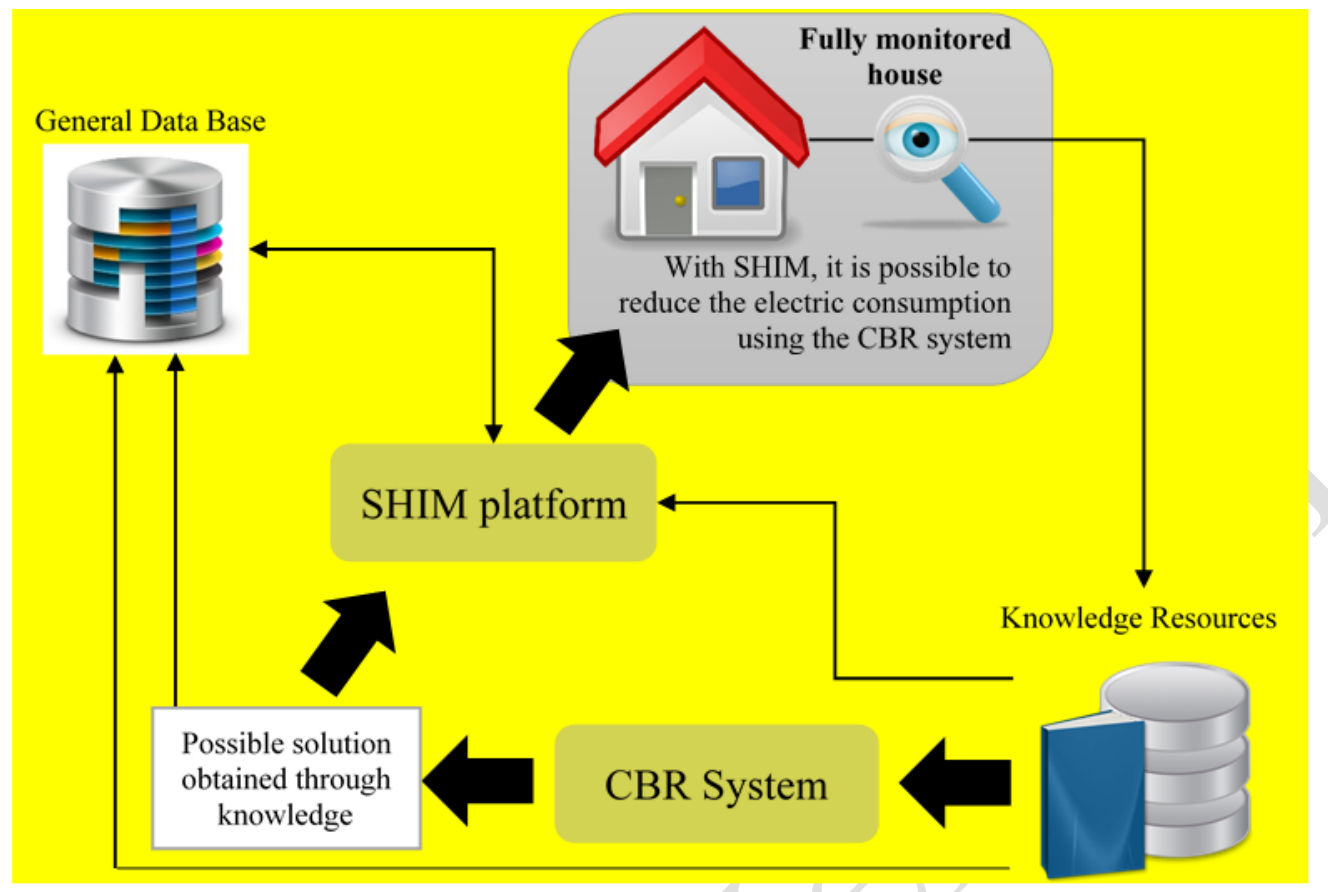

Figure 3 - Overview of the proposed model

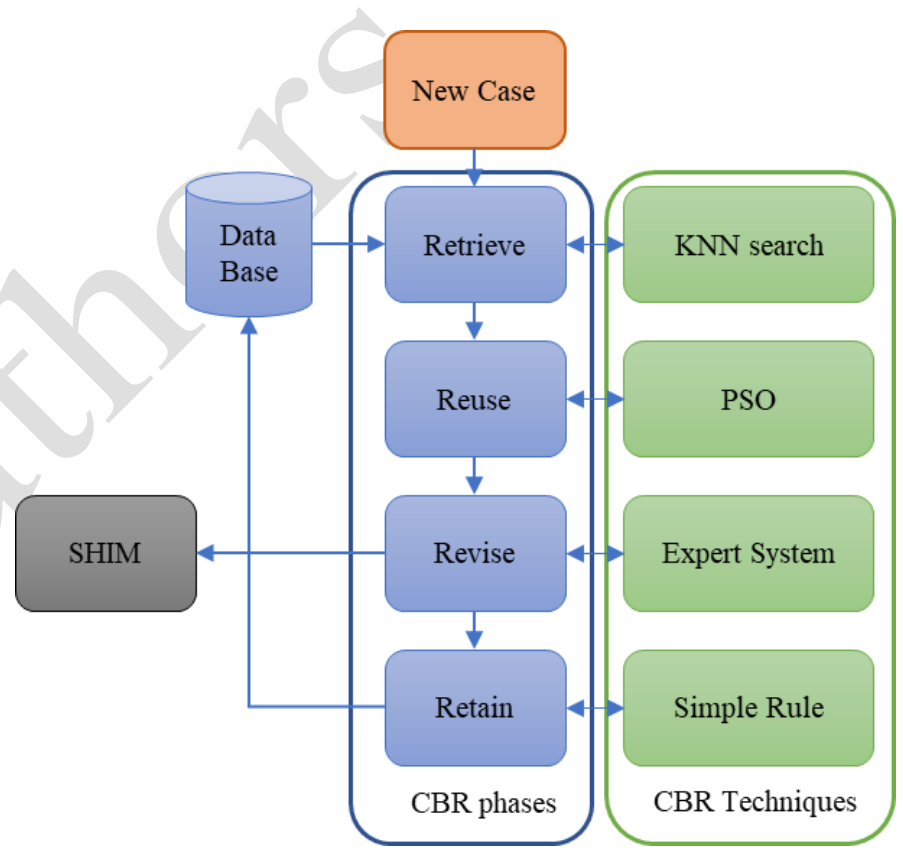

Figure 4 - Flowchart of the proposed CBR approach

As can be seen by Figure 4, the proposed CBR approach is composed by four steps, as described in detail in the following sections. During the retrieve phase, the past cases that are similar to the new case are identified 
and retrieved from the Data Base (DB). This is performed by applying the k-NN clustering algorithm. The main innovation of this work, apart from the scope of application, is the implementation of a PSO based approach to enable optimizing the importance of the different parameters that characterize each case for the calculation of the new result for the new case. The originated solution is then refined through the application of another innovative aspect of this work - an expert system that is composed by a set of ad-hoc rules that guarantee that the solution is adequate and applicable to the new case scenario. Finally, the retain phase determines if the new case should be included in the BD, according to its similarity to the cases that are already part of the BD.

In order to apply the proposed model, it is necessary to have a DB that inlcudes the historic provious cases. In this case, the available DB is constructed from the scenarios saved regarding the building for which the model will be applied [39]. This DB has 11 different variables, which are collected and recorded from different sensors and other types of data collection systems. TABLE I represents all of variables and their types.

TABLE I - Characteristics of variables used in the case.

\begin{tabular}{cccc}
\hline Representation & Name of variables & Variable type & Measurement scale (converted scale) \\
\hline$x_{1}$ & Weekday & Numerical & Integer \\
$x_{2}$ & Month & Numerical & Integer \\
$x_{3}$ & Hour & Hourly & Real Number \\
$x_{4}$ & Season & Numerical & Integer \\
$x_{5}$ & External Temperature & Numerical & Real Number \\
$x_{6}$ & External Humidity & Numerical & Real Number \\
$x_{7}$ & Persons Number & Numerical & Integer \\
$x_{8}$ & Electricity consumption & Numerical & Real Number \\
$x_{9}$ & Electricity Generation & Numerical & Real Number \\
$x_{10}$ & Electricity Tariff & Numerical & Real Number \\
$x_{n}$ & Electricity Reduction & Numerical & Rumber \\
\hline
\end{tabular}

As visible from TABLE I, $x_{1}$ is a variable that represents the day of the week. This variable enables the system to know in which day of the week the case occurs. In this variable, Sunday is equivalent to 1, Monday to 2 etc. until Saturday which is represented by 7. $x_{2}$ represents the month of the year, which is also represented by an integer value, where January corresponds to 1 continuing until December that is represented by a $12 . x_{3}$, 
represents the time to which the case refers. This variable undergoes a transformation from the time format to the numerical format (real number). $x_{4}$ represents the corresponding season of the year, where summer is equivalent to 1 , autumn to 2 , winter to 3 , and spring is equivalent to 4 . The external temperature $x_{5}$, external humidity $x_{6}$, electric consumption $x_{8}$, electric generation $x_{9}$ and the electricity tariff $x_{10}$, are considered as real numbers. The number of persons $x_{7}$ should be represented by an integer number, which in some cases, it may be zero. The electric reduction $R$, is the result of the association between the variables and is considered as the resolution of each case. While the system uses the CBR before a new case, a reduction value will be generated.

For creating the DB, it is necessary to normalize the values of the variables. The normalization process converts raw values to standart scores, which requires selecting the values that span one range and representing them in another range. Normalization is often done by dividing each value by the highest value recorded in the DB. This type of process can cause problems when it is working with a DB where there are variables of different natures and different ranges of values. This type of normalization is limited by the possibility of distorting the values of the different variables, since the DB variables can have large discrepancy between each other and they are different in their types, (e.g., binary, integer, etc.). In this work, the 11 types of variables consider diferent data ranges (presented in TABLE I), which are converted to a scale from 0 to 1 through a statistical standardization process. By assuming that the data are aproximated by the normal distribuation, this is converted to a standard normal distribuation, where the mean is 0 and the STandard Deviation (STD) is 1. Equation (2) presents the probability density function of the standard distribution.

$$
f(u)=\frac{1}{\sigma \sqrt{2 \pi}} e^{-\frac{1}{2}\left(\frac{u-\mu}{\sigma}\right)^{2}}
$$

Where:

- $f(u)$ represents the probability density of normal distribution;

- $\mu$ is the mean and is equal to 0 ;

- $\sigma$ is the standard division, and is equal to 1 ;

Equation (3) proposes the cumulative probability function, and is the function of each real number $u$. This is called cumulative distribution function, since it accumulates the probabilities values, which are less than $u$.

$$
F(u)=\sum_{u_{i} \leq x} f\left(u_{i}\right)
$$


Where:

- $\quad F(u)$ is the cumulative probability distribution function;

- $u_{i}$ represents the discrete random variable.

Equation (3) calculates the cumulative probability for each DB value, thus, it enables obtaining a DB with all values between 0 to 1 . In order to obtain the original values, it is simply necessary to apply the inverse function of the cumulative distribution.

\subsection{Task Retrieve}

As mentioned in section 2.1, the retrive step is the most important task of the CBR cycle and it is the task in which the system will select the most similar cases. In the proposed methodology, this process employs a $k$ nearest neighbor $(k-\mathrm{NN})$ technique [5], clustering based method [11], which is utilized to select the most similar cases. For this purpose, the $k$-NN algorithm uses a distance measure to analyse each case. This measure is the Euclidean distance and is expressed in equation (4).

$$
d\left(u_{i}, u_{j}\right)=\sqrt{\sum_{r=1}^{n}\left(\left(u_{i}\right)-\left(u_{j}\right)\right)^{2}}
$$

Where:

- $\quad n$ is the dimensionality of the input vector, namely the number of attributes of the examples;

- $\quad r$ is from 1 to $\mathrm{n}$;

When $d\left(u_{i}, u_{j}\right)$ becomes smaller, it means that the two examples are more similar. Equation (5) expresses the prediction that will be the class, and that has the most members in the k nearest neighbours.

$$
y\left(d_{i}\right)=\arg \max \sum_{u_{j} \in N N} y\left(u_{j}, c_{k}\right)
$$

Where:

- $d_{i}$ is a text example;

- $u_{j}$ is one of its $\mathrm{k}$ nearest neighbours in the training;

- $u_{j}, c_{k}$ indicates whether $u_{j}$ belongs to class $c_{k}$. 


\subsection{Task Reuse}

In the reuse task, a solution is obtained from the retrieved cases. In order to enable to arrive at a value equation (6) is used. According to Thomas Mitchell [23], this is called hypothesis fitness, and assumes that the electricity reduction $R$ of specific case $j$ can be formulated by appropriately weighting its attributes.

$$
R_{j}=w_{1} x_{1}+w_{2} x_{j 2}+w_{3} x_{j 3} \ldots+w_{i} x_{j i}
$$

Applying this relation to the set of cases obtained through the retrieved process, can be performed by equation (7).

$$
\left[\begin{array}{ccc}
x_{11} & \cdots & x_{1 i} \\
\vdots & \ddots & \vdots \\
x_{j 1} & \cdots & x_{i j}
\end{array}\right] \cdot\left[\begin{array}{c}
w_{1} \\
\vdots \\
w_{i}
\end{array}\right]=\left[\begin{array}{c}
R_{1} \\
\vdots \\
R_{j}
\end{array}\right]
$$

Equation (8) performs the matrix transformations where the vector of electricity reduction passes to the first member. In this equation, the matrix multiplication between the variable of each case with the weight of each variable subtracted from the electric reduction, is equal to a certain error $e_{j}$.

$$
\left[\begin{array}{ccc}
x_{11} & \cdots & x_{1 i} \\
\vdots & \ddots & \vdots \\
x_{j 1} & \cdots & x_{i j}
\end{array}\right] \cdot\left[\begin{array}{c}
w_{1} \\
\vdots \\
w_{i}
\end{array}\right]-\left[\begin{array}{c}
R_{1} \\
\vdots \\
R_{j}
\end{array}\right]=\left[\begin{array}{c}
e_{1} \\
\vdots \\
e_{j}
\end{array}\right]
$$

Equation (9) is an objective function that has the main goal of minimizing the sum of square root of the error $e_{j}$. The optimal combination of weight will be obtained when the function reaches a minimum value, which is gained by solving each one of the equations resulting from the matrix calculation. The number of equations will be equal to the number of retrieved cases.

$$
\min f(e)=\sqrt[2]{\sum_{\max j}^{j=1}\left(e_{j}\right)^{2}}
$$

For solving equation (9) and finding the ideal solution for combination of weights to minimize the equation, PSO is used $[12,40]$. There may not be a combination of weights that can achieve the global minimum, which would be 0 . This type of algoritm does not guarantee a global optimal solution; however, a exact methods are too time consuming, and would make the proposed too heavy to be applied as decision support for a fast response time; therefore a metaheuristic approach is required. Generally the search process in PSO is stopped when the stopping criterion is reached after repeating successively [7]. Equations (10) and (11) are applied to conduct the search. 


$$
\begin{gathered}
v_{i d}^{k+1}=w \cdot v_{i d}^{k}+c_{1} \cdot v_{1}^{k} \cdot\left(\text { Pbest }_{i d}^{k}-x_{i d}^{k}\right)+c_{2} \cdot r_{2}^{k} \cdot\left(\text { Gbest }_{i d}^{k}-x_{i d}^{k}\right) \\
x_{i d}^{k+1}=x_{i d}^{k}+v_{i d}^{k+1}
\end{gathered}
$$

Where:

- $\quad v_{i d}^{k}$ is the velocity of particle $i$, parameter $\mathrm{d}$ and iteration $k$;

- $\quad x_{i d}^{k}$ is position of particle $i$, parameter $\mathrm{d}$ and iteration $k$;

- $\quad k$ represents iteration;

- $\quad$ Pbest is personal best;

- Gbest stands for global best;

- $\quad w$ is inertia term;

- $\quad c_{1}$ presents local attraction term;

- $\quad c_{2}$ is global attraction term;

- $\quad r_{1}, r_{2}$ represents random numbers between $[0,1]$.

Equation (10) guides the algorithm search and equation (11) updates the new position that gives rise to a new solution. This solution is evaluated from the objective function expressed in equation (9). According to the literature, the search carried out by the PSO is very dependent on the factor of inertia $w$, since it controls the level of balance between the exploration and exploitation of the search space throughout the iterations. In this work, the inertia weight is defined by equation (12), and is proposed in [38].

$$
w_{k}=w_{\max }-\frac{\left(w_{\max }-w_{\min }\right)}{k_{\max }} \times k
$$

Where:

- $w_{\max }$ is the maximum value of inertia;

- $w_{\min }$ is the minimum value of inertia;

- $\quad k_{\max }$ is the maximum value of iterations.

After the PSO converges, the set of weights is obtained, and it is possible to apply these weights to the variables of the new case. Therefore, it obtains a reduction value, which corresponds to the result of the new case. 


\subsection{Task Revise}

In this work the revise task is formulated from the existing knowledge about the problem. It replicates an Expert System [35], which intends to emulate in CBR the decision-making ability of a human expert. For this purpose, the rules presented in this sub-section have been created, which are applied to the solution obtained by the reuse task. The main target of the created rules is variable $x_{3}$ representing the hour of the day. In equation (13), if the result of reuse task (CRreuse) is less than zero, the result of the revise task (CRrevise) will be zero.

$$
\text { if CRreuse }<0 \rightarrow \text { CRrevise }=0
$$

Equation (14) defines the rule for the hours between 0 and 5. In this rule, it is imposed on the system that at least there must be $C_{\min } \mathrm{kW}$ of consumption, and reduce the remaining by half. $x_{8}$ indicates the electric consumption in this and other equations.

$$
\text { if } x_{3} \geq 0 \cap x_{3}<5 \cap\left(\text { CRreuse }=0 \cup x_{8}>\text { Cmin }\right) \rightarrow \text { CRrevise }=\frac{x_{8}-\text { Cmin }}{2}
$$

Equation (15) represents the meal hours. At this time, the reduction should be $25 \%$ of the value corresponding to the load $x_{8}$, less the production itself, represeting by $x_{9}$ in the equations.

$$
\begin{aligned}
\text { if }\left(\left(x_{3}>7.3 \cap x_{3}<9\right) \cup\left(x_{3}>12 \cap x_{3}<13.3\right) \cup\left(x_{3}>19.3 \cap x_{3}<21\right)\right) \\
\cap\left(\text { CRreuse }=0 \cup \text { CRreuse }>x_{8}-x_{9}\right) \rightarrow \text { CRrevise }=0.25 \times\left(x_{8}-x_{9}\right)
\end{aligned}
$$

Equation (16) represents the hours between the breakfast and lunch, and from lunch to dinner. $x_{7}$ represents the number of inhabitants, which is taken into account by this rule, and if it is less than 3 , the value of the reduction will be half of the consumption, to which the production is substracted; otherwise the reduction will be a quarter.

$$
\begin{gathered}
\text { if }\left(\left(x_{3}>9 \cap x_{3}<12\right) \cup\left(x_{3}>14 \cap x_{3}<18\right)\right) \cap\left(\text { CRreuse }=0 \cup \text { CRreuse }>x_{8}-x_{9}\right) \\
\text { elseif } x_{7}<3 \rightarrow \text { CRrevise }=\frac{x_{8}-x_{9}}{2} \\
\text { else CRrevise }=\frac{x_{8}-x_{9}}{4}
\end{gathered}
$$

Equation (17) represents the hours between 21 of the current day and 0 of the next day. The value of the reduction is half of the load minus the production, however, it is expected that the residential production in this schedule is around zero.

$$
\text { if } x_{3}>21 \cap x_{3}<0 \cap\left(\text { CRreuse }=0 \cup \text { CRreuse }>x_{8}-x_{9}\right) \rightarrow \text { CRrevise }=\frac{x_{8}-x_{9}}{2}
$$


Equation (18) represents the hours between 5 and 7.3, and it is defined so that the 24 hours are all covered. This rule indicates a reduction of $10 \%$ in all consumption above the minimum standard consumption $C_{\min }$.

$$
\text { if } \begin{aligned}
x_{3}>5 \cap x_{3}< & 7.3 \cap\left(\text { CRreuse }=0 \cup x_{8}>\text { Cmin }\right) \rightarrow \text { CRrevise } \\
& =0.8+0.1 \times\left(x_{8}-\text { Cmin }\right)
\end{aligned}
$$

At the end of this task, when all defined rules are applied to the results of the reuse task, the revised value will be obtained.

\subsection{Task Retain}

This is the last task of the cycle, which decides if the new case should or not be incorporated in the DB. For this goal, equations (19) and (20)are expressed.

$$
\frac{\left|S H I M_{\text {result }}-C R_{\text {result }}\right|}{S H I M_{\text {result }}} \leq 0.2
$$

In equation (19), one of the conditions that the new case should respect to be incorporated in the DB, is represented. If the difference between the SHIM result and the CBR result is greater than 0.2 , the new case will be excluded, since it doe not represent a good enough solution (the error is too big).

$$
\text { Similarity }\{\text { new case, best similar case in } D B\} \leq 95 \%
$$

According to equation (20), only new cases with a similarity smaller than $95 \%$ relatively to the most similar case within DB will be accepted in DB. This is defined so that the DB is not filled with a numerous number of very similar cases, which do not added new valuable information. If these two conditions (equation (19) and (20)) are true, the new case is incorporated in the DB.

\section{Case study}

This section describes the case study that is carried out in order to demonstrate the performance of the proposed methodology. Two sub-sections are presented; the first one assesses the performance of the proposed CBR methodology by comparing the CBR results with some reference a-priori known cases. The second subsection considers the evaluation of the proposed approach using the SHIM BEMS. In specific, the solution provided by the proposed methodology for a completely new case is sent to SHIM, so that this system may schedule the reduction of loads according to the output of the CBR method. This way it is possible to assess the impact of the results on the users' comfort, and if it is, in fact, feasible to apply these results in a real application scenario. 
This case study considers a standard house with 4 inhabitants, located in Porto, northern Portugal. All loads of this house are completely emulated in a laboratorial setting in GECAD/ISEP campus. All loads are equipped with monitoring and control. Lighting devices are equipped with ballasts controlled by one of two different technologies: analog signal input; and DALI protocol. The building management system provides a 0-10 V analog signal in order to control the actual percentage lighting level. Using the DALI protocol, the building management system is able communicate the actual lighting level to the balast. Additionally, one generic Programmable Logic Controller (PLC) per room is able to accommodate both control means. However, although this case study is applied in this controlled house in order to enable the analysis of the proposed methodology application impact, the results of the proposed approach can be applied to any other house. If the application house is equipped with monitoring and control means, the outputs from the energy management can be applied directly in the loads; on the other hand, if the house does not include such equipment, the results are provided to the user by means of suggestions, which the user may apply manually. The consumption profile of each load is dependent on several factors, such as the time, day, season, temperature, etc. The detailed consumption profile of the house devices for a specific day is shown in sub-section 5.2, in order to assess the impact of the proposed methodology for a specific case. TABLE II shows the characteristics of the different loads considered in the house.

As TABLE II demonstrates, the considered house includes several loads, with different consumption values. It should be noted that the microwave and the oven are the loads that have the higher consumption in this scenario. The aggregation of these different loads in a given period of the day, provides the total consumed energy in that period. A set of previous cases regarding the used loads at each time, the contextual information and the reduction applied (as described in TABLE I) is used to feed the CBR DB. TABLE II also shows the possibility that each home appliance has in its control, which is crucial for the energy management process, because there are cases where it will be impossible to act since it would significantly reduce the user's comfort. The non-controllable devices are the home appliances in which the control of the consumption values is not possible. This means that they are either disconnected or connected. The controllable devices are those in which it is possible to adjust their consumption, making it possible to reduce or increase the consumption value. Finally, shiftable devices are those whose consumption can be changed to other periods in time. 
TABLE II - Characteristics of home appliances.

\begin{tabular}{ccccc}
\hline Home appliance & Power $(\mathbf{K W})$ & \multicolumn{2}{c}{ Controlling options } & \\
& & Non-controllable & Controllable & Shiftable \\
\hline Microwave & 1,2 & $\mathrm{X}$ & $\mathrm{X}$ \\
Oven & 1,5 & $\mathrm{X}$ & $\mathrm{X}$ \\
Coffee machine & 0,6 & $\mathrm{X}$ & $\mathrm{X}$ \\
Refrigerator 1 & 0,1 & $\mathrm{X}$ & $\mathrm{X}$ \\
Refrigerator 2 & 0,12 & $\mathrm{X}$ & $\mathrm{X}$ \\
Washing machine & 0,8 & $\mathrm{X}$ & $\mathrm{X}$ & $\mathrm{X}$ \\
HVAC & 0,6 & & $\mathrm{X}$ & \\
Light hall & 0,06 & & $\mathrm{X}$ & \\
Light room & 0,06 & & $\mathrm{X}$ & \\
Light kitchen & 0,12 & & $\mathrm{X}$ & \\
Light living room & 0,1 & $\mathrm{X}$ & & \\
TV room & 0,138 & 0,124 & & $\mathrm{X}$ \\
TV living room & 0,3 & & \\
Water heater & & & & \\
\hline
\end{tabular}

\subsection{CBR performance}

Five cases from the DB are considered as the subject cases - they are extracted from the BD and are no longer considered as previous cases, so that the proposed methodology can be applied and the achieved results compared to the original real values that have been registered for these cases. The selection was made taking into account the time of the day $\left(x_{3}\right)$, in order to consider cases that refer to different hours. The five considered cases are shown in TABLE III. 
TABLE III - Profile of subject cases for model validation

\begin{tabular}{cccccccccccc}
\hline Case & $\boldsymbol{x}_{\mathbf{1}}$ & $\boldsymbol{x}_{\mathbf{2}}$ & $\boldsymbol{x}_{\mathbf{3}}$ & $\boldsymbol{x}_{\mathbf{4}}$ & $\boldsymbol{x}_{\mathbf{5}}$ & $\boldsymbol{x}_{\mathbf{6}}$ & $\boldsymbol{x}_{\mathbf{7}}$ & $\boldsymbol{x}_{\mathbf{8}}$ & $\boldsymbol{x}_{\mathbf{9}}$ & $\boldsymbol{x}_{\mathbf{1 0}}$ & $\boldsymbol{R}_{\text {real }}$ \\
\hline Case 1 & 7 & 1 & 2,45 & 3 & 11,2 & 95 & 4 & 0,6 & 0 & 0,1634 & 0 \\
Case 2 & 1 & 8 & 9,30 & 1 & 17,5 & 86 & 4 & 0,12 & 0,98 & 0,1634 & 0 \\
Case 3 & 1 & 5 & 12,15 & 4 & 20,7 & 66 & 4 & 3,211 & 1,6 & 0,1634 & 0 \\
Case 4 & 7 & 10 & 18,45 & 2 & 20,2 & 89 & 4 & 3,435 & 0,125 & 0,1634 & 0,05 \\
Case 5 & 7 & 10 & 23,45 & 2 & 20,6 & 67 & 6 & 2,413 & 0 & 0,1634 & 0,09 \\
\hline
\end{tabular}

The validation of the CBR model is done, in a first instance, from the comparison of the real values and the values proposed by the CBR. Each of the presented cases is considered as a new case, therefore, the proposed CBR model is executed 5 times. In each cycle, the CBR receives the referent case applies the retrieve task, which selects the most similar cases in the DB. After this task, the set of weights for each variable is calculated in order to be applied to the variables that compose the vase, thus originating the reduction value proposed by CBR. After the revise process, where the solution is reviewed, the retention process can only be carried out when SHIM completes its action. SHIM is applied using the value of reduction proposed by the CBR approach, in order to assess if such reduction is feasible to be applied to the set of loads that re being used in each case, without compromising the users' comfort.

In order to analyse the impact of $k$ (number of nearest neighbours - most similar cases) selected for the reuse task, different options are experimented, namely: considering all cases in the $\mathrm{DB}, k=10, k=5, k=3$, and $k=1$. Due to the influence of the PSO in the optimization objective function - equation (9), two experiences are performed: The first one considers 10 executions of the PSO and the second considers 100 cycles. TABLE IV, presents the results of proposed methodology, represented through the value of Root Mean Square Error (RMSE) between the CBR output value and the real reduction value from the original cases, and the mean execution time. The presented values consider the average (error and time) of the 5 considered subject cases, and the time is counted from the presentation of the new case to the presentation of a solution. 
TABLE IV - Proposed CBR methodology results

\begin{tabular}{ccccc}
\hline \multirow{2}{*}{$\boldsymbol{c}$ 10 PSO Runs } & \multicolumn{2}{c}{ 100 PSO Runs } \\
& RMSE & Time Mean (s) & RMSE & Time Mean (s) \\
\hline All cases & 0,067786035 & 13,11114527 & 0,060142167 & 99,0616876 \\
10 & 0,118635 & 11,92354007 & 0,065897729 & 87,22773526 \\
5 & 0,056884244 & 12,00484601 & 0,051008033 & 85,54363879 \\
3 & 0,084792965 & 11,96009029 & 0,043725463 & 84,42065277 \\
1 & 0,08213981 & 9,747374915 & 0,031159179 & 63,82456464 \\
\hline
\end{tabular}

By analysing the value of the RMSE shown in TABLE IV, one can verify that when 10 executions of the PSO are performed, the achieved value is subjected to a large randomness and therefore it is not possible to establish a connection between the selected number of $k$ and the achieved RMSE. It is noticeable that the highest RMSE value was recorded for $k=5$. Regarding the execution time value for the 10 execution of PSO, it is possible to verify that there is a decreasing trend when a smaller $k$ is considered.

When 100 executions of the PSO are executed, it is possible to establish a relation between the $k$ and the RMSE value. Although with $k=10$, the RMSE value is greater than the selection of all the cases, there is a clear tendency of decreasing RMSE when a smaller $k$ is considered. This indicates that it is better for the CBR process to consider less, but more similar cases, than considering a large number of less similar cases. This decreasing trend is also verified regarding the execution time, which is due to the smaller amount of data to process. When comparing the execution time of the 10 execution with the 100 executions, it is possible to verify an increase when considering more executions, since there are 10 times more executions carried out with the PSO. Figure 5 illustrates the details in terms of execution times for the case of $k=5$.

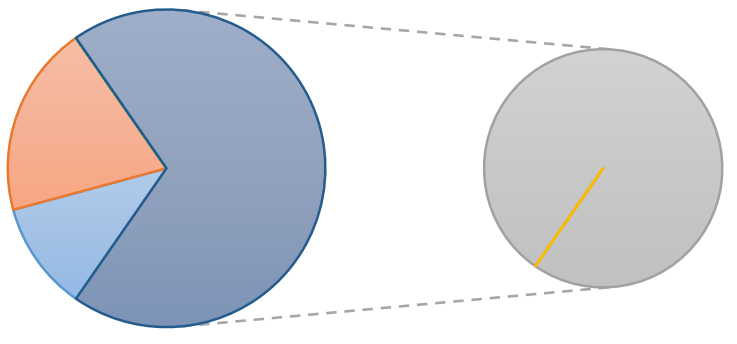

$\square$ Load $\square$ Retrieve $\square$ PSO $\square$ Adaptation $\square$ Revise $\square$ Retain

(a)

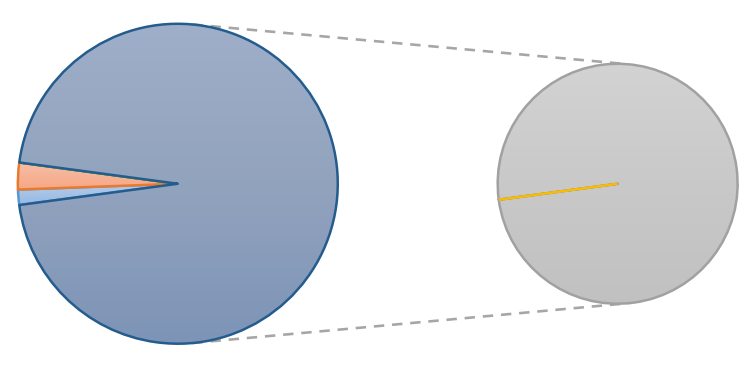

$\square$ Load $\square$ Retrieve $\square$ PSO $\square$ Adaptation $\square$ Revise $\square$ Retain

(b)

Figure 5 - Execution time when considering $k=5$, a) 10 PSO cycles, b) 100 PSO cycles 
The results presented in Figure 5 refer to the mean values for all executions considering $k=5$. In this figure, the 4 stages of the CBR cycle and the loading of the data are represented. The reuse phase is not expressed in the legend of Figure 5, since it is constituted by the PSO phase. In Figure 5 a), the total time is 12 seconds, and in Figure 5 b) it is 86 seconds. This significant difference is due to the time that the PSO requires to search for the solution. The values of the revise and retain tasks are residual in percentage terms and in both simulations, they reach less than $0.1 \%$ of the time.

In Figure 5 a), the optimization time of the PSO takes about $69 \%$ of the time, while the retrieve task and the load takes respectively $19 \%$ and $11 \%$ of the time. In Figure $5 \mathrm{~b}$ ) the percentages for the optimization time of the PSO, retrieve, and load are respectively $95 \%, 2,7 \%$ and $1,7 \%$ of the time. When comparing the PSO times, the 100 executions take nearly 10 times more. The time for 10 cycles is 11,92 seconds, and for 100 cycles is 87,23 second.

TABLE V shows the obtained values from the CBR for each case as well as the real value and the recorded error. These results are presented for the two optimization scenarios of 10 and 100 PSO executions.

TABLE V - Results for $k=5$

\begin{tabular}{|c|c|c|c|c|c|c|}
\hline \multirow{2}{*}{ Case } & \multicolumn{3}{|c|}{10 PSO executions } & \multicolumn{3}{|c|}{100 PSO executions } \\
\hline & $\boldsymbol{R}_{C R}$ & $\boldsymbol{R}_{\text {real }}$ & $\mid$ Error $\mid$ & $R_{C R}$ & $\boldsymbol{R}_{\text {real }}$ & $\mid$ Error $\mid$ \\
\hline Case 1 & 0,027637 & 0 & 0,02763711 & 0,016333 & 0 & 0,016333 \\
\hline Case 2 & $0 *$ & 0 & 0 & $0 *$ & 0 & 0 \\
\hline Case 3 & 0,170157 & 0 & 0,17015733 & 0,143535 & 0 & 0,143535 \\
\hline Case 4 & 0,236603 & 0,05 & 0,18660306 & 0,222625 & 0,05 & 0,172625 \\
\hline Case 5 & 0,217896 & 0,09 & 0,12789607 & 0,209904 & 0,09 & 0,119904 \\
\hline
\end{tabular}

*Resulting from review at the revise stage.

As TABLE V shows, the error that occurs between the reduction value proposed by CBR and the actual value decreases when 100 executions of PSO are performed. This means the PSO search highly influences the final value of reduction.

In order to assess the relation between $k$ and the objective function value, subject case 5 is analysed in detail, as shown in TABLE VI. 
TABLE VI - Results of PSO optimization for subject case 5

\begin{tabular}{ccccccc}
\hline PSO executions & Measurements & $\boldsymbol{k}=$ All cases & $\boldsymbol{k}=\mathbf{1 0}$ & $\boldsymbol{k}=\mathbf{5}$ & $\boldsymbol{k}=\mathbf{3}$ & $\boldsymbol{k}=\mathbf{1}$ \\
\hline \multirow{3}{*}{10} & Minimum & 0,430837 & 0,166229 & 0,126317 & 0,182658 & 0,000517 \\
& Mean & 1,922872 & 0,621862 & 0,4466 & 0,481716 & 0,128692 \\
& STD & 1,092854 & 0,517035 & 0,232452 & 0,218454 & 0,136354 \\
& Minimum & 0,454242 & 0,164527 & 0,124212 & 0,090836 & 0 \\
100 & Mean & 2,194597 & 0,835225 & 0,42778 & 0,368762 & 0,207027 \\
& STD & 1,468844 & 0,568206 & 0,299163 & 0,289947 & 0,159124 \\
\hline
\end{tabular}

From TABLE VI one can conclude that when $k$ decreases, the objective function approaches 0 , which is normal since the objective function becomes easier to solve, since there is less noise from least similar cases. Furthermore, as TABLE VI shows, if 100 PSO executions are executed by the system, a better value of objective function is obtained.

Figure 6 illustrates the results of k-NN algorithm for subject case 1. As expected, the k-NN makes the selection regarding the similarity between the cases. The representations are only in $2 \mathrm{D}$, but the selection is performed considering the 10 variables.

According to the different sections of Figure 6, as k is reduced, the k-NN algorithm selects the most similar cases. In this case, the reduction value of the different cases is represented. TABLE VII shows the indices of the selected cases, which correspond to the selected cases visible in Figure 6. As expected, the most similar cases are repeated until the selection is made for $\mathrm{k}=1$. In this context, case 11 has the most similarity to case 1 . From TABLE VII it is visible that the k-NN technique is efficient in the selection of most similar cases. From TABLE VII it is visible that, as expected, the most similar cases are repeated until the selection is made for $\mathrm{k}=1$. In this context, case 11 has the most similarity to case 1 . From TABLE VII it is visible that the k-NN technique is efficient in the selection of most similar cases. 


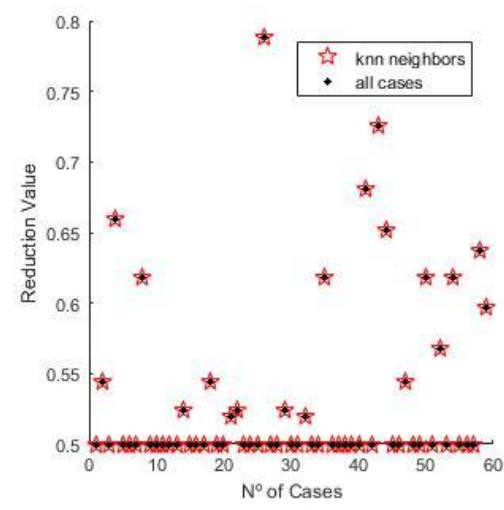

(a)

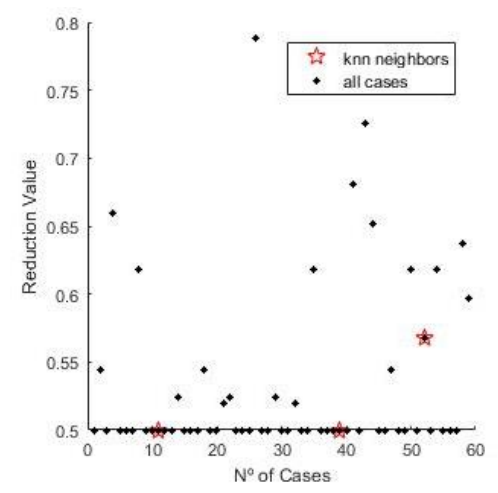

(d)

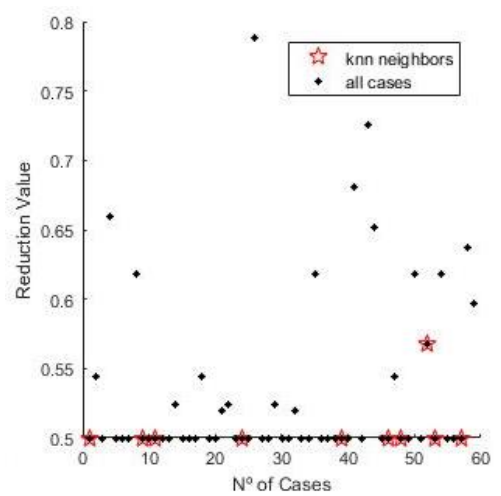

(b)

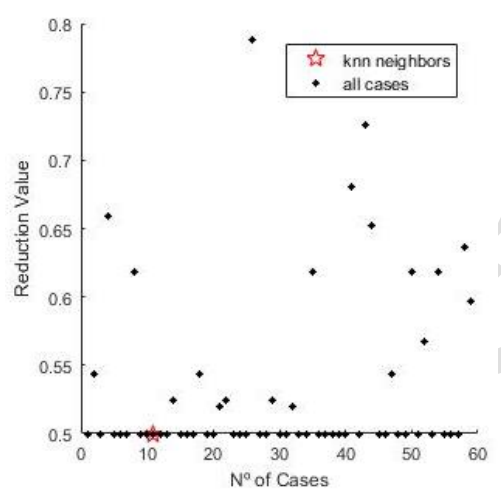

(e)

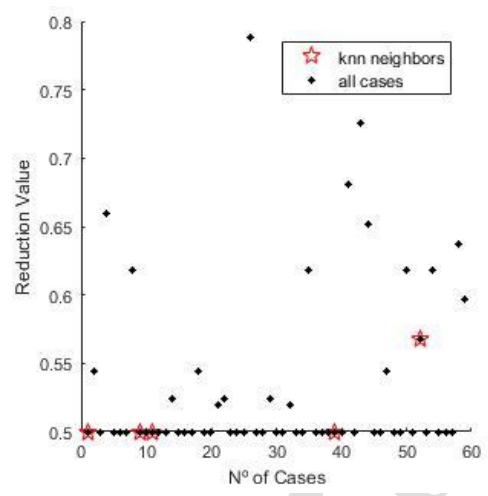

(c)

Figure $6-\mathrm{k}-\mathrm{NN}$ selection of cases for subject case 1: a) $k=$ all cases, b) $k=10$, c) $k=5$, d) $k=3 \mathrm{~m}$ and e) $k=1$

TABLE VII - Similar cases selected for case 1

\begin{tabular}{cc}
\hline$k$ & Index of case \\
\hline All cases & All cases \\
10 & $\{1,9,11,24,39,46,48,52,53$ and 57$\}$ \\
5 & $\{1,9,11,39$ and 52$\}$ \\
3 & $\{11,39$ and 52$\}$ \\
1 & $\{11\}$ \\
\hline
\end{tabular}

\subsection{CBR evaluation using SHIM}

In order to validate the proposed model using SHIM, a specific simulation day is considered. This considered day is a Tuesday in July. Figure 7 shows the total consumption of the house throughout the 24 hours of the considered simulation day. Additionally, this figure also shows the amount of consumption reduction that is suggested by the proposed methodology throughout the day. 


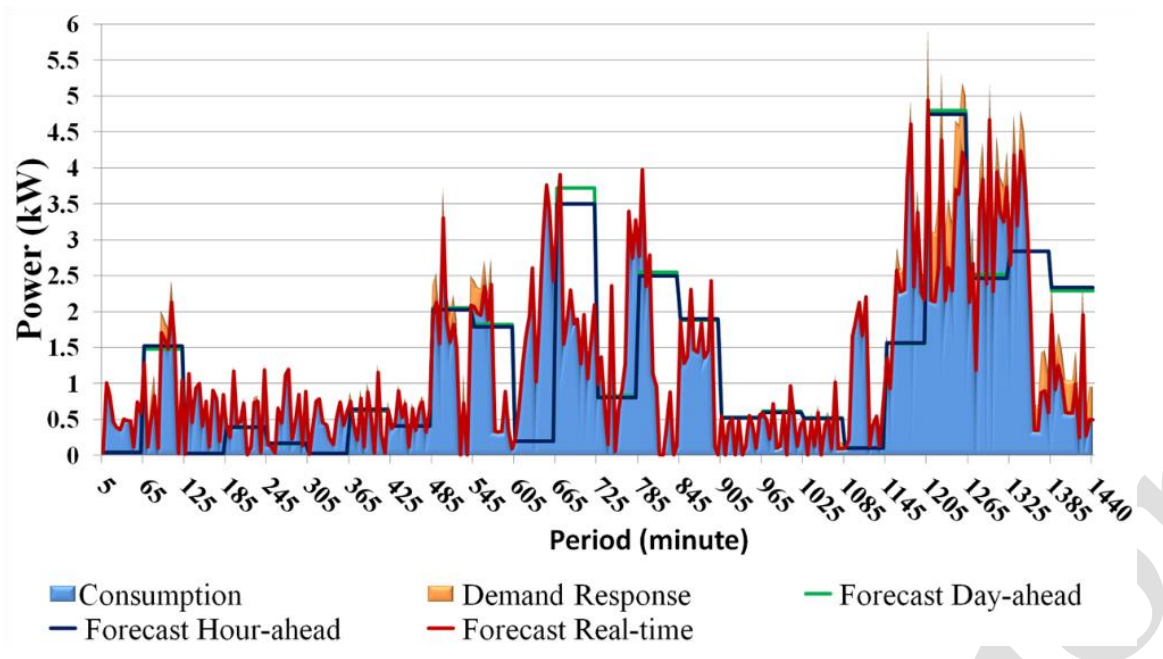

Figure 7 - Total consumption of the house throughout the considered day, and demand response reduction amount resulting from the proposed methodology

From Figure 7 it is visible that some amounts of reduction (identified as demand response in the figure) are suggested by the proposed methodology to some periods of the day, especially during the peak hours of consumption. Figure 8 shows the results of SHIM when applying the reductions resulting from the proposed method to the several devices of the house. The red line represents the consumption limit resulting from the proposed methodology to be applied by the Energy Resource Management (ERM) performed by SHIM. Hence, all consumption values above the line are amounts being reduced.

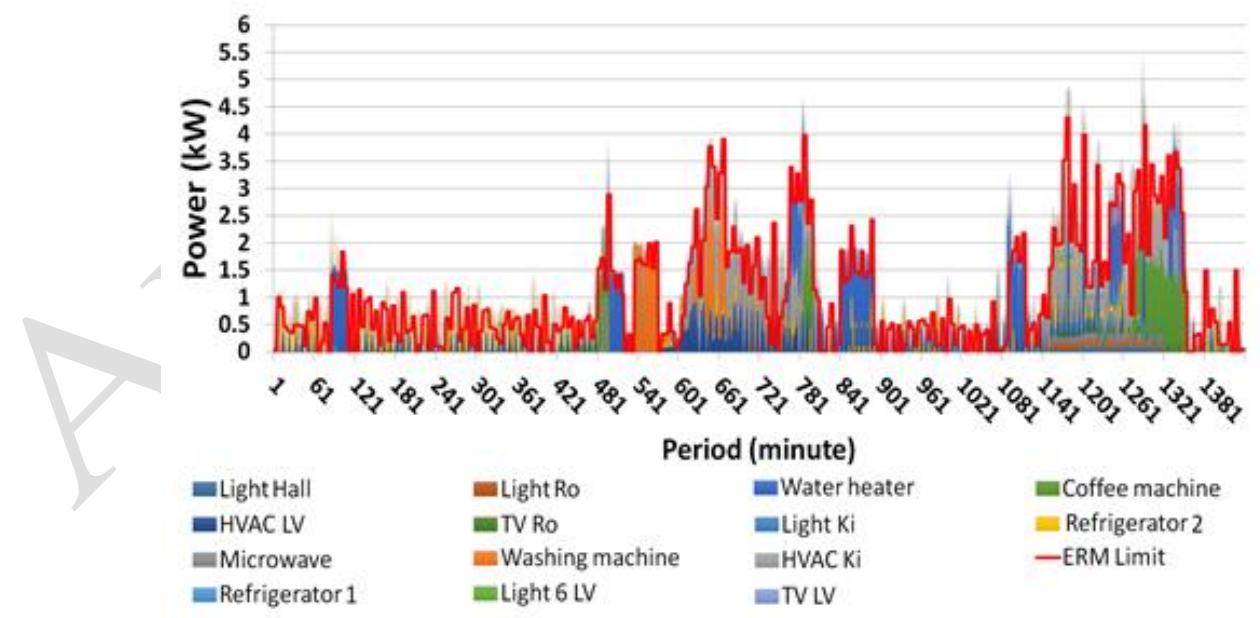

Figure 8 -Consumption per device of the house throughout the considered day, and reduction applied to each device 
From Figure 8 it is visible that SHIM scheduled the consumption of the several devices according to the reduction values provided the proposed methodology.

In order to analyse the performance of the proposed methodology, and the impact of SHIM over these results, a specific case is considered, where the application of the proposed methodology is explained in detail. This case considers a specific time during the considered day, namely at 7 p.m., when all 4 inhabitants are present inside the house. The variables of this case are shown in Table VIII. With these input variables, the CBR model is able to select the most similar cases and present a reduction value that will be the input for SHIM.

Table VIII - Test case variables characterization

\begin{tabular}{cccccccccccc}
\hline Case & $\boldsymbol{x}_{\mathbf{1}}$ & $\boldsymbol{x}_{\mathbf{2}}$ & $\boldsymbol{x}_{\mathbf{3}}$ & $\boldsymbol{x}_{\mathbf{4}}$ & $\boldsymbol{x}_{\mathbf{5}}$ & $\boldsymbol{x}_{\mathbf{6}}$ & $\boldsymbol{x}_{\mathbf{7}}$ & $\boldsymbol{x}_{\mathbf{8}}$ & $\boldsymbol{x}_{\mathbf{9}}$ & $\boldsymbol{x}_{\mathbf{1 0}}$ & $\boldsymbol{R}_{\text {real }}$ \\
\hline New Case & 3 & 7 & 19 & 1 & 14 & 85 & 4 & 1,779 & 0,05 & 0,1634 & - \\
\hline
\end{tabular}

Five simulations are done, which result in five different values of reduction, as can be seen in Table IX. The average reduction from the five simulations is also presented as one of the solutions. These five solutions are based on the value of similar cases selected in the retrieve process. In the SHIM algorithm, the possible reduction value is limited by the decimal places so given this fact it is only possible to obtain a reduction value with two decimal places. In Table IX the input values of reduction for SHIM algorithm are presented.

Table IX - SHIM inputs

\begin{tabular}{cccc}
\hline & Expected Reduction $(\mathrm{kW})$ & Reduction $(\mathrm{kW})$ & Difference $(\mathrm{kW})$ \\
\hline$k=$ All cases & 0,18596002 & 0,17 & 0,015960017 \\
$k=10$ & 0,17969616 & 0,17 & 0,009696158 \\
$k=5$ & 0,16759278 & 0,17 & $-0,002407225$ \\
$k=3$ & 0,16348578 & 0,16 & 0,003485777 \\
$k=1$ & 0,05001877 & 0,05 & $1,87651 \mathrm{E}-05$ \\
Mean & 0,1493507 & 0,15 & $-0,000649302$ \\
\hline
\end{tabular}

As Table IX shows, the values of reduction are limited, and the maximum reduction value according to the user comfort, from SHIM is $0,17 \mathrm{~kW}$. If the reduction is too high (in this scenario above $0,17 \mathrm{~kW}$ ), it decreases the comfort level of the occupants. As can be seen from the Table IX the best presented reduction values are the results of $k=5$ and $k=3$, for three reasons: (i) these have a very small difference to the actual reduced value, 
(ii) these enable performing a larger reduction than when considering $k=1$, and (iii) SHIM is able to respect the suggested reduction without compromising the user comfort, contrarily to the results of $k=10$ and $k=$ all cases, which suggest values of reduction that are higher than the maximum level of reduction without affecting the comfort $-0,17 \mathrm{~kW}$. Figure 9 shows the appliances that are consuming energy in the considered test case. As one can see in Figure 9, only four equipment are connected at the considered time and are consuming 1,779 kW in total. Making the analogy with TABLE II one can see that there are ten equipment turned off. The appliances that are consuming have a load higher than $95 \%$ of their maximum consumption.

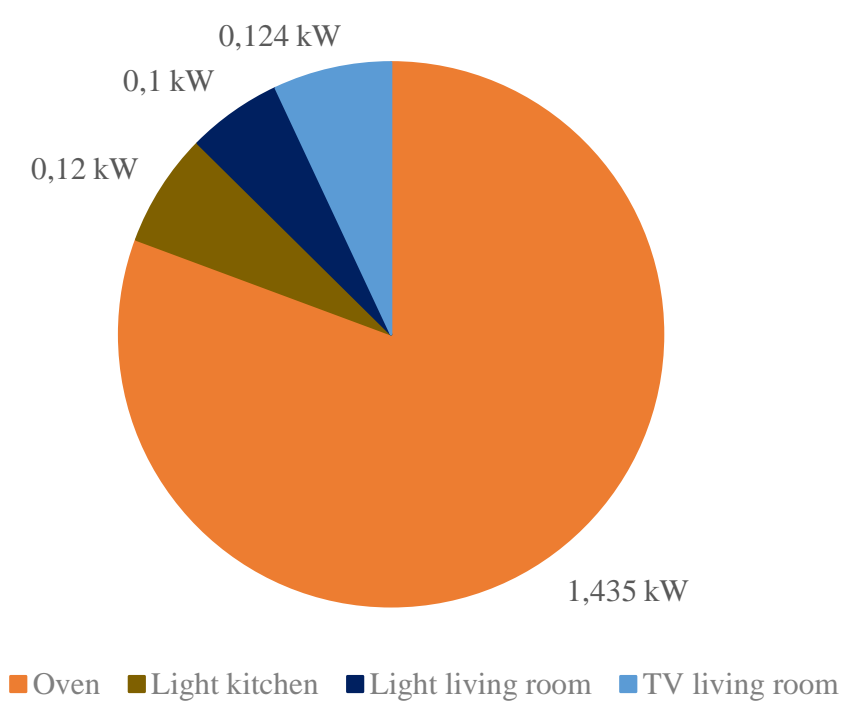

Figure 9 - Consumption profile of the four connected appliances.

By analysing the new case of Table VIII, it is a pre-dinner scenario, where the occupants are in the kitchen to cook (oven and kitchen lights connected), while others wait for dinner in the living room (television and living room lights connected). It is also a day in the month of June with an exterior temperature of 14 degrees. Therefore, it is not necessary to consume energy for the house's refreshment; hence the system of cooling is off.

Figure 10 represents the reduction results for all reduction values resulting from the several simulations of the CBR model. The consumption profiles resulting from the imposed reduction are also shown in Figure 10 . The consumption profile prior to the reduction was presented (Figure 9), therefore, it is possible to make the comparison between the both. 


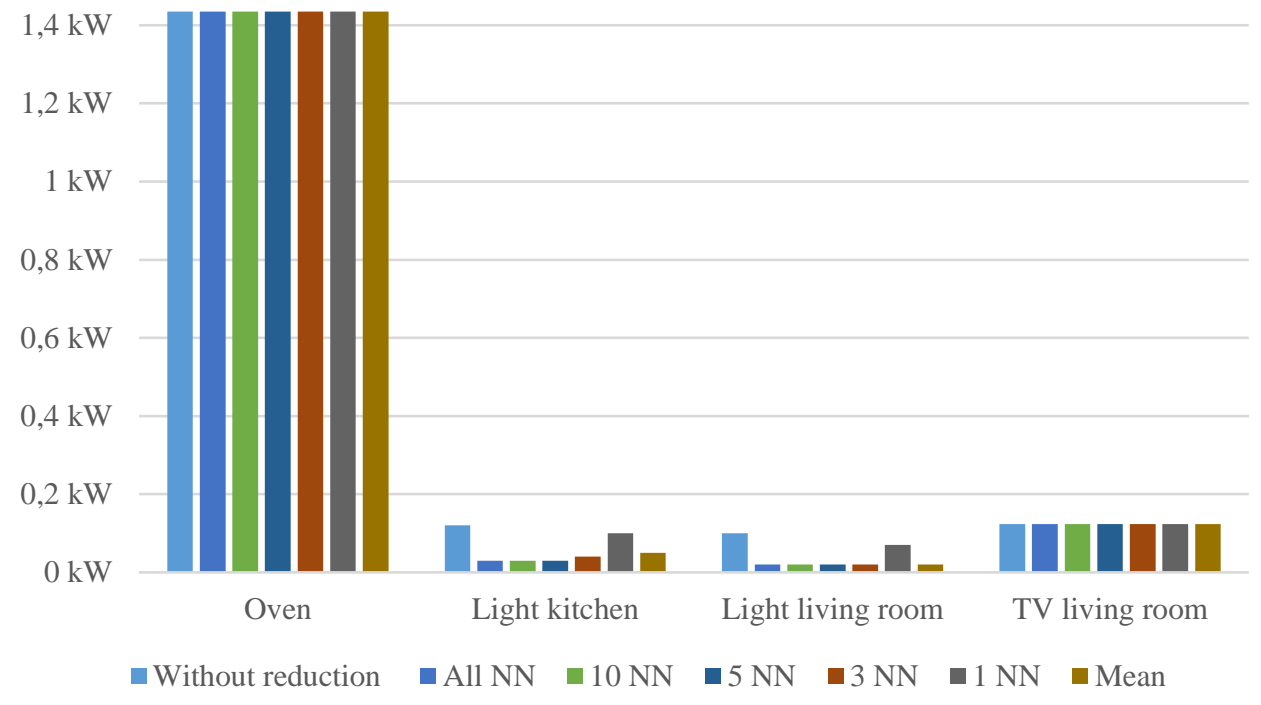

Figure 10 - Consumption of equipment in the various scenarios

Figure 10 only shows the equipment that were turned on. As it is possible to observe, in the oven no reduction was imposed, which is reasonable since any change could cause to spoil cooking, and consequently reduce the inhabitants comfort level. This scenario is also true for the TV located in the living room. TV is a device whose consumption cannot be reduced. This means that it can be turned on and its consumption is on maximum, or it is turned off and its consumption is zero. In this context, the lights are the only equipment available for reduction. For this purpose, it is necessary that the building has enough infrastructure to execute the reduction (for instance, the lights equipped with some intelligent ballasts or dimmer). As it can be observed on Figure 10, the consumption of the lights has been reduced while the rest of connected equipment stay without any changes in their consumption.

For $k=$ all cases, $k=10 \mathrm{NN}$ and $k=5$, the kitchen lights suffer a reduction of $0.09 \mathrm{~kW}$ and living room lights are reduced by $0.08 \mathrm{~kW}$. For $k=3,0.08 \mathrm{~kW}$ is the reduction applied to both equipment. For the case of $k=1, \mathrm{a}$ smaller reduction occurs, in which the kitchen lights suffer a reduction of $0.02 \mathrm{~kW}$ and living room lights suffer $0.03 \mathrm{~kW}$. In the mean case, the kitchen lights had $0.07 \mathrm{~kW}$ of reduction and the living room lights suffer 0.08 $\mathrm{kW}$.

The reduction that occurred was $9.6 \%$ in maximum, which is a small reduction. However, by performing these types of operation at any instant of time, it will be possible to have economic savings for the user and also 
determine the best way of responding to demand response events. Additionally, it is noticeable that the comfort level of the user should always be taken into account.

\section{Conclusions}

This paper presented a CBR methodology to obtain suggested reduction values for house energy management. This methodology presents an innovative combination of two different approaches: intelligent house management and CBR, which provides a new body of knowledge regarding the current inexistence of such approach in the literature. The proposed approach enables the possibility of adjusting the instant consumption of a house according to the required reduction values in each moment. The CBR approach can find the solution of reduction for instant consumption profile without large computational efforts, which is by itself another relevant contribution. SHIM is able to apply the reduction in real houses, by applying the required reduction in different home appliances according to their priority regarding the needs and comfort of the users. The CBR DB can bring a limitation of this methodology, because many cases with similarity between them is needed to reach good results. On the other hand, the existing cases in the DB should cover every possible option or at least similar cases, which is also difficult to verify, especially in early stages of the management and execution. Another limitation is the fact the implementation of CBR systems is generally very specific and problem-directed. Thus, the application of this model in other areas, or slightly different problems, requires significant changes. On the other hand, this combination is able to present an instant reduction result in a short

time, which a mathematical model cannot achieve due to the number of included variables and difficulty in modelling all the necessary constraints, and more importantly, having access to all the required information in real time.

The proposed CBR methodology considers the application of the k-NN algorithm in the retrieve phase, the optimization of weights related to importance of each variable that composes a case for the similarity analysis, using PSO, and an expert system for the revise phase. The outputs from the proposed methodology are used by the energy resource management system SHIM.

The k-NN technique proved that is efficient to select the most similar cases. The performance of the PSO approach has been assessed, considering 10 and 100 executions, from which resulted the conclusion that the final results are very dependent on the optimization performed by PSO. Furthermore, as more cycles are performed, the most precise results PSO provides. It is also concluded that the selection of many similar cases 
can spoil the final solution. When the final results of the system and the actual values are compared, it can be concluded that the lower the number of similar cases offers fewer errors. It is also visible that the reduction values that result from the proposed methodology are appropriate, as validated by the execution of the case in SHIM, which has showed that the reductions are feasible without compromising the users' comfort.

As future work different techniques of case selection will be experimented, such as the decision trees. In the reuse task, it may also be advantageous to use different techniques to optimize the weights associated to each variable that composes a case, such as genetic algorithms and simulated annealing.

\section{Acknowledgements}

This work is supported by FEDER Funds through COMPETE program and by National Funds through FCT under the projects FCOMP-01-0124-FEDER: PEst-OE/EEI/UI0760/2015; PTDC/SEN-ENR/122174/2010; and PTDC/EEA-EEL/122988/2010.

\section{References}

[1] A. Aamodt, E. Plaza, Case-Based Reasoning: Foundational Issues, Methodological Variations, and System Approaches, AI Commun. IOS Press. 7 (1994) 39-59.

[2] R. Bareiss, PROTOS; a unified approach to concept representation, classification and learning, University of Texas at Austin, 1988.

[3] C. Bolchini, A. Geronazzo, E. Quintarelli, Smart buildings: A monitoring and data analysis methodological framework, Build. Environ. 121 (2017) 93-105.

[4] J.L. Castro, M. Navarro, J.M. Sánchez, J.M. Zurita, Loss and gain functions for CBR retrieval, Inf. Sci. (Ny). 179 (2009) 1738-1750.

[5] Z. Deng, X. Zhu, D. Cheng, M. Zong, S. Zhang, Efficient kNN classification algorithm for big data, Neurocomputing. 195 (2016) 143-148.

[6] A.I. Dounis, C. Caraiscos, Advanced control systems engineering for energy and comfort management in a building environment—A review, Renew. Sustain. Energy Rev. 13 (2009) 1246-1261.

[7] R. Eberhart, J. Kennedy, A new optimizer using particle swarm theory, Micro Mach. Hum. Sci. 1995. MHS 95., Proc. Sixth Int. Symp. (1995) 39-43.

[8] European Commission, DIRECTIVE 2010/31/EU OF THE EUROPEAN PARLIAMENT AND OF THE COUNCIL of 19 May 2010 on the energy performance of buildings (recast), 2010. 
[9] European Commission, Directive 2010/31/EU of the European Parliament and of the Council of 19 May 2010 on the energy performance of buildings, 2010.

[10] European Commission, Challenges ahead - Research and Innovation - Key Enabling Technologies, (2017).

[11] R. Faia, T. Pinto, Z. Vale, Dynamic Fuzzy Clustering Method for Decision Support in Electricity Markets Negotiation, ADCAIJ Adv. Distrib. Comput. Artif. Intell. J. 5 (2016) 23.

[12] R. Faia, T. Pinto, Z. Vale, E.J.S. Pires, Portfolio Optimization for Electricity Market Participation with Particle Swarm, Fourth Int. Work. Artif. Intell. Tech. Power Syst. Energy Mark. (IATEM 2015) 26th Int. Conf. Database Expert Syst. Appl. (DEXA 2015). (2015) 62-67.

[13] F. Fernandes, H. Morais, Z. Vale, C. Ramos, Dynamic load management in a smart home to participate in demand response events, Energy Build. 82 (2014) 592-606.

[14] K.H. Im, S.C. Park, Case-based reasoning and neural network based expert system for personalization, Expert Syst. Appl. 32 (2007) 77-85.

[15] M. Imran, R. Hashim, N.E.A. Khalid, An Overview of Particle Swarm Optimization Variants, Procedia Eng. 53 (2013) 491-496.

[16] J. Iwaro, A. Mwasha, A review of building energy regulation and policy for energy conservation in developing countries, Energy Policy. 38 (2010) 7744-7755.

[17] J. Kolodner, Case-based Reasoning, Morgan Kaufmann Publishers Inc., San Francisco, CA, USA, 1993.

[18] D.B. Leake, D.C. Wilson, When Experience is Wrong: Examining CBR for Changing Tasks and Environments ?,(1999).

[19] R. Lopez De Mantaras, D. Mcsherry, D. Bridge, D. Leake, B. Smyth, S. Craw, B. Faltings, M. Lou Maher, M. Cox, K. Forbus, M. Keane, A. Aamodt, I. Watson, Retrieval, reuse, revision and retention in case-based reasoning, Knowl. Eng. Rev. 20 (2005) 215.

[20] M. Manic, D. Wijayasekara, K. Amarasinghe, J.J. Rodriguez-Andina, Building Energy Management Systems: The Age of Intelligent and Adaptive Buildings, IEEE Ind. Electron. Mag. 10 (2016) 25-39.

[21] J. McCarthy, The Frame Problem Today, in: Fram. Probl. Artif. Intell., Morgan Kaufmann Publishers, Stanford, 1987: p. 3. 
[22] R. Missaoui, H. Joumaa, S. Ploix, S. Bacha, Managing energy Smart Homes according to energy prices: Analysis of a Building Energy Management System, Energy Build. 71 (2014) 155-167.

[23] T.M. Mitchell, Machine Learning, 1st ed., McGraw-Hill, Inc., New York, NY, USA, 1997.

[24] P. Oliveira, T. Pinto, H. Morais, Z. Vale, MASGriP a multi-agent smart grid simulation platform, IEEE Power Energy Soc. Gen. Meet. (2012) 1-8.

[25] Y.-J. Park, B.-C. Kim, S.-H. Chun, New knowledge extraction technique using probability for casebased reasoning: application to medical diagnosis, Expert Syst. 23 (2006) 2-20.

[26] R. Platon, V.R. Dehkordi, J. Martel, Hourly prediction of a building's electricity consumption using case-based reasoning, artificial neural networks and principal component analysis, Energy Build. 92 (2015) 10-18.

[27] C. Rodrigues, F. Freire, Adaptive reuse of buildings: Eco-efficiency assessment of retrofit strategies for alternative uses of an historic building, J. Clean. Prod. 157 (2017) 94-105.

[28] J.W. Schaaf, Fish and Shrink. A next step towards efficient case retrieval in large scaled case bases, in: I. Smith, B. Faltings (Eds.), Adv. Case-Based Reason. Third Eur. Work. EWCBR-96 Lausanne, Switzerland, Novemb. 14--16, 1996 Proc., Springer Berlin Heidelberg, Berlin, Heidelberg, 1996: pp. $362-376$.

[29] R.C. Schank, Dynamic Memory: A Theory of Reminding and Learning in Computers and People, Cambridge University Press, New York, USA, 1983.

[30] I. Sharma, J. Dong, A.A. Malikopoulos, M. Street, J. Ostrowski, T. Kuruganti, R. Jackson, A modeling framework for optimal energy management of a residential building, Energy Build. 130 (2016) 55-63.

[31] K. Shin, I. Han, Case-based reasoning supported by genetic algorithms for corporate bond rating, Expert Syst. Appl. 16 (1999) 85-95.

[32] A.G. De Silva Garza, M. Lou Maher, A Process Model for Evolutionary Design Case Adaptation, in: J.S. Gero (Ed.), Artif. Intell. Des. '00, Springer Netherlands, Dordrecht, 2000: pp. 393-412.

[33] N. Soares, J. Bastos, L.D. Pereira, A. Soares, A.R. Amaral, E. Asadi, E. Rodrigues, F.B. Lamas, H. Monteiro, M.A.R. Lopes, A.R. Gaspar, A review on current advances in the energy and environmental performance of buildings towards a more sustainable built environment, Renew. Sustain. Energy Rev. 77 (2017) 845-860. 
[34] M.M. Veloso, J.G. Carbonell, Case-Based Reasoning in Prodigy, in: R.S. Michalski, G. Teccuci (Eds.), Mach. Learn. A Multistrategy Approach, Vol. IV, Morgan Kaufmann, 1994: pp. 523-548.

[35] W.P. Wagner, Trends in expert system development: A longitudinal content analysis of over thirty years of expert system case studies, Expert Syst. Appl. 76 (2017) 85-96.

[36] Z. Wang, R.S. Srinivasan, A review of artificial intelligence based building energy use prediction: Contrasting the capabilities of single and ensemble prediction models, Renew. Sustain. Energy Rev. 75 (2017) 796-808.

[37] X. Xiao, M. Skitmore, X. Hu, Case-based Reasoning and Text Mining for Green Building Decision Making, Energy Procedia. 111 (2017) 417-425.

[38] J. Xin, G. Chen, Y. Hai, A Particle Swarm Optimizer with Multi-stage Linearly-Decreasing Inertia Weight, Comput. Sci. Optim. 2009. CSO 2009. Int. Jt. Conf. 1 (2009) 505-508.

[39] Z.J. Yu, F. Haghighat, B.C.M. Fung, Advances and challenges in building engineering and data mining applications for energy-efficient communities, Sustain. Cities Soc. 25 (2016) 33-38.

[40] W. Zhang, J. Liu, L. Fan, Y. Liu, D. Ma, Control strategy PSO, Appl. Soft Comput. 38 (2016) 75-86. 